\title{
A Green Bank Telescope Search for Water Masers in Nearby Active Galactic Nuclei
}

\section{Citation}

Braatz, J. A., C. Henkel, L. J. Greenhill, J. M. Moran, and A. S. Wilson. 2004. "A Green Bank Telescope Search for Water Masers in Nearby Active Galactic Nuclei." The Astrophysical Journal 617 (1) (November 11): L29-L32. doi:10.1086/427185. http://dx.doi.org/10.1086/427185.

\section{Published Version}

$10.1086 / 427185$

\section{Permanent link}

http://nrs.harvard.edu/urn-3:HUL.InstRepos:32085452

\section{Terms of Use}

This article was downloaded from Harvard University's DASH repository, and is made available under the terms and conditions applicable to Other Posted Material, as set forth at http:// nrs.harvard.edu/urn-3:HUL.InstRepos:dash.current.terms-of-use\#LAA

\section{Share Your Story}

The Harvard community has made this article openly available.

Please share how this access benefits you. Submit a story.

\section{Accessibility}




\title{
A GBT Search for Water Masers in Nearby AGNs
}

\author{
J. A. Braatz \\ National Radio Astronomy Observatory ${ }^{1}$, 520 Edgemont Rd., Charlottesville, VA 22903 \\ C. Henkel \\ Max-Planck-Institut für Radioastronomie, Auf dem Hügel 69, D-53121 Bonn, Germany \\ L. J. Greenhill² and J. M. Moran \\ Harvard-Smithsonian Center for Astrophysics, 60 Garden Street, Cambridge, MA 02138 \\ A. S. Wilson ${ }^{3}$ \\ Department of Astronomy, University of Maryland, College Park, MD 20742
}

\begin{abstract}
Using the Green Bank Telescope, we have conducted a survey for $1.3 \mathrm{~cm}$ water maser emission toward the nuclei of nearby active galaxies, the most sensitive large survey for $\mathrm{H}_{2} \mathrm{O}$ masers to date. Among 145 galaxies observed, maser emission was newly detected in eleven sources and confirmed in one other. Our survey targeted nearby $\left(\mathrm{v}<12,000 \mathrm{~km} \mathrm{~s}^{-1}\right)$, mainly type 2 AGNs north of $\delta=-20^{\circ}$, and includes a few additional sources as well. We find that more than a third of Seyfert 2 galaxies have strong maser emission, though the detection rate declines beyond $\mathrm{v} \sim 5000 \mathrm{~km} \mathrm{~s}^{-1}$ due to sensitivity limits. Two of the masers discovered during this survey are found in unexpected hosts: NGC 4151 (Seyfert 1.5) and NGC 2782 (starburst). We discuss the possible relations between the large X-ray column to NGC 4151 and a possible hidden AGN in NGC 2782 to the detected masers. Four of the masers discovered here, NGC 591, NGC 4388, NGC 5728 and NGC 6323, have high-velocity lines symmetrically spaced about the systemic velocity, a likely signature of molecular gas in a nuclear accretion disk. The maser
\end{abstract}

\footnotetext{
${ }^{1}$ The National Radio Astronomy Observatory is a facility of the National Science Foundation operated under cooperative agreement with Associated Universities, Inc.

${ }^{2}$ Current address: Kavli Institute for Particle Astrophysics and Cosmology, Stanford Linear Accelerator Center, 2575 Sand Hill Rd., Menlo Park, CA 94025, USA

${ }^{3}$ Adjunct Astronomer, Space Telescope Science Institute, Baltimore, MD 21218
} 
source in NGC 6323, in particular, reveals the classic spectrum of a "disk maser" represented by three distinct groups of Doppler components. Future single-dish and VLBI observations of these four galaxies could provide a measurement of the distance to each galaxy, and of the Hubble constant, independent of standard candle calibrations.

Subject headings: galaxies: active — galaxies: nuclei — galaxies: Seyfert — ISM: molecules — masers — radio lines: galaxies

\section{Introduction}

Water vapor masers form in warm $(300-1000 \mathrm{~K})$, dense $\left(10^{7}-10^{11} \mathrm{~cm}^{-3}\right)$ gas and are common in the envelopes of late-type stars and in star-forming regions. They have been detected throughout the Milky Way and in many external galaxies as well. Some extragalactic water masers are simply analogues to the masers seen in star-forming clouds in our own galaxy. Others are found in more exotic locations, especially the accretion disks and gaseous outflows associated with active galactic nuclei (AGN). Owing to their large apparent isotropic luminosity, and following the terminology applied to strong extragalactic $\mathrm{OH}$ masers, those $\mathrm{H}_{2} \mathrm{O}$ masers detected in active galactic nuclei have been called "megamasers". Review articles on the topic of water megamasers have been written recently by Maloney (2002), Greenhill (2002) and Henkel and Braatz (2003).

At $1.3 \mathrm{~cm}$ wavelength, water masers can be imaged with mas resolution using VLBI. Maps of masers in the nearby Sy 2/LINER nucleus of NGC 4258 (Greenhill et al. 1995b; Miyoshi et al. 1995; Herrnstein et al. 1999) showed that they arise in a thin, warped, edgeon disk at a galactocentric radius of $0.14-0.28$ pc. Maser components near the systemic velocity form in clouds on the near side of the disk, and "satellite lines" with velocities up to $\pm 1100 \mathrm{~km} \mathrm{~s}^{-1}$ (Humphreys et al. 2003) from systemic form in gas located at points viewed tangentially to its orbital path. The maser disk in NGC 4258 follows a Keplerian rotation curve and implies a central mass of $3.9 \times 10^{7} \mathrm{M}_{\odot}$ (Herrnstein et al. 1999). The satellite lines also reveal the angular size of the disk as well as its shape (i.e. the warp).

The velocities of maser lines near the systemic velocity in NGC 4258 drift redward through a $\sim 100 \mathrm{~km} \mathrm{~s}^{-1}$ window at a rate of about $9 \mathrm{~km} \mathrm{~s}^{-1} \mathrm{yr}^{-1}$ (Haschick, Baan \& Peng 1994; Greenhill et al. 1995a). The drift reveals the centripetal acceleration of gas in the disk as it moves across our line of sight to the central core. Herrnstein et al. (1999) developed a model of the maser disk based on its VLBI structure, and combined their derived disk parameters with the measured acceleration to calculate the linear size of the 
disk. Comparing the linear size of the disk with its angular size, they determined a distance to the galaxy independent of the usual use of standard candles. The authors also used the independent technique of proper motions to confirm the result. Establishing a set of distances to other galaxies using these techniques would provide a new foundation to the extragalactic distance ladder uncoupled from other methods that have historically involved large systematic uncertainties.

There has been a resurgent interest in surveys for extragalactic water maser sources in recent years, inspired by both the above issues and new instrumentation. Programs by Hagiwara, Diamond and Miyoshi (2002), Henkel et al. (2002), Greenhill et al (2003), Hagiwara et al. (2003), Kondratko et al. (2003), and Peck et al. (2003), among others, have increased the number of known extragalactic $\mathrm{H}_{2} \mathrm{O}$ masers to $\sim 50$. Still, only a few of these masers have the signature of a nuclear disk, and more examples are needed.

In this paper we discuss the results of a new survey for $\mathrm{H}_{2} \mathrm{O}$ maser emission from a sample of nearby $\left(\mathrm{v}<12,000 \mathrm{~km} \mathrm{~s}^{-1}\right)$ galaxies, nearly all AGNs. With the goal of maximizing the detection rate and searching for disk maser candidates, we concentrated on type 2 Seyferts and LINERs, which are known to be the most likely hosts of detectable masers (Braatz, Wilson \& Henkel 1997). The candidates were primarily selected from the CfA Seyfert Catalog (Huchra, available at http://cfa-www.harvard.edu/ huchra), a collection of AGNs drawn from CfA redshift surveys and identifications of X-ray and infrared sources. Eight galaxies in our survey are not in this catalog but were of interest because of strong infrared luminosity or known nonstellar nuclear activity. These are IC 694, IC 4553, NGC 3660, NGC 4013, NGC 5635, NGC 5953, NGC 6211, and UGC 3995A.

Each of the galaxies observed during the present survey has been observed in at least one previous survey for water vapor maser emission, e.g. by Braatz, Wilson \& Henkel (1996), Greenhill et al. (1997) or Taylor et al. (2002). The motivation to reobserve these sources comes primarily from recent advances in K-band sensitivity, especially due to the availability of the Robert C. Byrd Green Bank Telescope (GBT). In addition, the availability of large bandwidth $(>200 \mathrm{MHz}$ ) spectrometer modes now makes it possible to search efficiently for high-velocity lines. Finally, the intrinsic variability of $\mathrm{H}_{2} \mathrm{O}$ masers makes it worthwhile to reobserve sources even if they have been previously undetected. Galaxies already known to host $\mathrm{H}_{2} \mathrm{O}$ masers were not observed during this survey, but are being studied as part of other programs, e.g. Braatz et al. (2003). 


\section{Observations}

Observations were made with the GBT during several sessions between 2003 March 4 and 2004 February 28. This period marked a steady improvement in the GBT's high frequency observing capabilities, particularly with regard to baseline stability, pointing accuracy, focus tracking, and sensitivity. We used the $18-22 \mathrm{GHz}$ K-band receiver, which has two beams at a fixed separation of $3^{\prime}$ in azimuth. The GBT beam width is $\sim 36^{\prime \prime}$ at $22 \mathrm{GHz}$, and pointing uncertainties were $3^{\prime \prime}-8^{\prime \prime}$. The telescope was nodded between two positions on the sky such that the source was always in one of the two beams during integration. Prior to 2003 June 4, we observed with the electronic beam switch in the receiver cycling at a rate of $1 \mathrm{~Hz}$, and used a nod cycle of 5 minutes. After that date, we observed with no electronic beam switching, and we shortened the nod cycle to 2 minutes. The latter configuration provided equally good baselines, a simpler data format, and better system reliability since a sometimes faulty electronic switch was bypassed.

The spectrometer was configured with two $200 \mathrm{MHz}$ bandpasses, one centered on the systemic velocity of the galaxy and the second redshifted by $180 \mathrm{MHz}$. Channel spacing in the spectra corresponds to $0.33 \mathrm{~km} \mathrm{~s}^{-1}$. The zenith system temperature was between $35 \mathrm{~K}$ and $80 \mathrm{~K}$. Atmospheric opacity was either measured with tipping scans or estimated from system temperature and weather data, and ranged from 0.04 to 0.12 at the zenith. Data were reduced using AIPS++. When baseline stability permitted, we smoothed the reference spectra prior to calibration using a 16 channel boxcar function, to improve sensitivity. In most cases we subtracted a polynomial (order between 3 and 6) from the $200 \mathrm{MHz}$ spectrum to remove the baseline shape. For some galaxies we subtracted a baseline structure derived from the lowest frequency Fourier components of the spectrum. The $1 \sigma$ r.m.s. sensitivity of the survey ranges between 3 and 6 mJy per $24.4 \mathrm{kHz}\left(\sim 0.33 \mathrm{~km} \mathrm{~s}^{-1}\right)$ channel. This sensitivity is equivalent to roughly $0.5 L_{\odot}$ for a $1 \mathrm{~km} \mathrm{~s}^{-1}$ line in a galaxy with a recession velocity of $5000 \mathrm{~km} \mathrm{~s}^{-1}$.

Velocities throughout this paper follow the optical definition of Doppler shift and are calculated with respect to the Local Standard of Rest (LSR). Galaxy recession velocities are derived either from $21 \mathrm{~cm}$ HI observations or optical observations. Galaxy distances are calculated using $\mathrm{H}_{0}=75 \mathrm{~km} \mathrm{~s}^{-1} \mathrm{Mpc}^{-1}$.

\section{Results and Discussion}

We observed 145 nearby AGNs during the survey, and detected masers in 12 galaxies (Table 1), giving a detection rate of $8.3 \%$. Without our knowledge at the time of the 
observation, one of these masers (in Mrk 1066) had been detected at Effelsberg by Peck et al (2003); the other 11 are new discoveries. Maser emission has been detected in each of the 12 galaxies with the GBT on at least two epochs, and the best spectrum for each one is shown in Figure 1. A complete presentation of data and an analysis of the variability in these sources will be presented in a later publication. The total number of galaxies, excluding the Milky Way, with detected $\mathrm{H}_{2} \mathrm{O}$ masers is now 63. Table 1 lists the galaxies detected during this survey and Table 2 lists those observed but not detected. Each galaxy was observed toward its nucleus. No maser emission was detected in any of the galaxies in the second bandpass, shifted by $180 \mathrm{MHz}$. A more comprehensive list of galaxies observed for $\mathrm{H}_{2} \mathrm{O}$ maser emission, including this survey and others, is available from http://www.nrao.edu/ jbraatz, and a list of known extragalactic maser systems is available at the site as well.

The galaxies observed during this survey do not form a complete sample but the results can still be used to improve our knowledge of the incidence of detectable masers in AGNs. Among galaxies listed as Seyfert 2 type in the CfA AGN list (see Sect. 1) and observed with the GBT (either during the present survey or during previous observations of known $\mathrm{H}_{2} \mathrm{O}$ masers, e.g. Braatz et al. 2003), the fraction detected is $17 / 82=21 \%, 14 / 55=25 \%$, and $11 / 39=38 \%$ for galaxies within $12,000 \mathrm{~km} \mathrm{~s}^{-1}, 7500 \mathrm{~km} \mathrm{~s}^{-1}$, and $5000 \mathrm{~km} \mathrm{~s}^{-1}$, respectively. Such a decrease of detection rate with increasing distance reflects the sensitivity limitation of surveys such as this (see Braatz, Wilson \& Henkel 1997). In the listed distance categories, there were 15, 9, and 2 galaxies, respectively, from the CfA list not observed during this survey. The LINER sample is less complete than the Seyfert 2 sample, so likewise statistics are less instructive for this class of AGN. Still we note that the detection rate is $5 / 42=$ $12 \%, 5 / 40=13 \%$, and $4 / 36=11 \%$ for LINERs from the present survey in the same three distance categories.

Two of the sources detected in this survey, NGC 2782 and NGC 4151, are included in Carl Seyfert's original list of twelve "Emission Line Galaxies" (Seyfert, 1943) that defined the Seyfert type, and it is interesting to note that five of those twelve have now been detected in $\mathrm{H}_{2} \mathrm{O}$ maser emission. However, NGC 2782 and NGC 4151 are not usually classified as type 2 AGNs, counter to the trend of most other extragalactic $\mathrm{H}_{2} \mathrm{O}$ masers. NGC 2782 has a powerful nuclear starburst but the high luminosity of the water masers $\left(\mathrm{L}_{i s o}=12 L_{\odot}\right)$ suggests the presence of an AGN. Kennicutt, Keel \& Blaha (1989) find that the profiles of the $\mathrm{H} \beta$ and [OIII] $\lambda \lambda$ 4959, 5007 lines are dramatically different, with [OIII] showing both a broader core and high velocity wings. They conclude that the high velocity gas in the wings of [OIII] must have very high excitation, reminiscent of the narrow line emission in Seyfert galaxies, and that the nucleus may be a "composite" (i.e. starburst plus AGN) system (cf. Véron, Gonçalves \& Véron-Cetty 1997). Other workers (e.g. Schulz et al. 1998) have, however, interpreted the high excitation gas in terms of a starburst-driven superwind, so the 
evidence for an AGN in NGC 2782 is not compelling. A hard X-ray spectrum of this galaxy could settle the issue.

NGC 4151 is a nearby (13.3 Mpc) active galaxy usually classified as a Seyfert 1.5 (e.g., Osterbrock \& Koski 1976), but the broad emission lines in its optical spectrum are variable (e.g. Sergeev, Pronik \& Sergeeva 2001) giving it a Seyfert 2 profile at times. The galaxy has a large X-ray column density toward its nucleus $\left(\sim 10^{23} \mathrm{~cm}^{-2}\right.$, Weaver et al. 1994). The proximity of NGC 4151 and this large X-ray column are conducive to the detection of water vapor emission. Braatz et al. (1997) concluded that the probability of $\mathrm{H}_{2} \mathrm{O}$ maser emission in Seyfert galaxies increases with increasing X-ray inferred column density to the nucleus. The X-ray measured column density to the nucleus of NGC 4151 is roughly two orders of magnitude larger than that toward a typical Seyfert 1, and is similar to the column found among typical Seyfert 2s (Turner et al. 1997). At $0.7 L_{\odot}$, the isotropic luminosity of the maser in NGC 4151 is among the weakest of any detected in an AGN. The maser emission is primarily confined to two narrow components (see Figure 1), one at $692.4 \mathrm{~km} \mathrm{~s}^{-1}$ and the other at $1126.6 \mathrm{~km} \mathrm{~s}^{-1}$. The widths of the emission lines, determined from Gaussian fits, are 1.2 and $1.5 \mathrm{~km} \mathrm{~s}^{-1}$ (full width at half maximum), respectively. NGC 4151 has been searched previously for $\mathrm{H}_{2} \mathrm{O}$ emission, but not detected. The most sensitive previous observation, obtained on 2000 March 20, resulted in a $3 \sigma$ detection limit of 9 mJy per $4.2 \mathrm{~km} \mathrm{~s}^{-1}$ channel (Taylor et al. 2002). Our detection shows the lines having peak flux densities of 36 and 53 mJy (Figure 1), but the lines are narrower than the channel spacing used by Taylor et al. The peak flux densities in our spectrum are reduced to $\sim 11$ and $\sim 19$ mJy after averaging to a channel spacing of $4.2 \mathrm{~km} \mathrm{~s}^{-1}$. That these fluxes are still above the Taylor et al. detection limit may reflect intrinsic variability in the NGC 4151 maser.

Three sources detected in this survey are associated with merging systems: NGC 2782, NGC 4922, and NGC 5256. In NGC 4922 the separation of the merging nuclei is $22^{\prime \prime}$ (10 $\mathrm{kpc}$ ) and each nucleus is itself an AGN. NGC 5256 is similar, with two AGNs in the merger separated by $11^{\prime \prime}(5.9 \mathrm{kpc})$. In both systems, an observation was made toward each of the nuclei to determine the source of the $\mathrm{H}_{2} \mathrm{O}$ emission. In NGC 4922 the observations are consistent with all of the detected maser emission originating from component NGC $4922 \mathrm{~N}$ (the more northern component) and in NGC 5256 the observations are consistent with all of the detected maser emission originating in NGC 5256S (the more southern component).

The masers detected in NGC 591, NGC 4388, NGC 5728 and NGC 6323 all reveal high-velocity features approximately symmetrically spaced about the systemic velocity of the galaxy, a possible signature of a nuclear disk. In this interpretation, the maximum rotation velocities would be $\sim 425, \sim 400, \sim 250$ and $\sim 550 \mathrm{~km} \mathrm{~s}^{-1}$ respectively. Two of these galaxies, NGC 4388 and NGC 5728, are known to have a well defined, bi-conically shaped 
narrow line region (Pogge 1988; Corbin, Baldwin \& Wilson 1988; Wilson et al. 1993), a characteristic feature of a highly inclined, optically thick nuclear torus around the source of ionizing radiation. NGC 6323 has the classic maser spectrum expected from a nuclear disk, with a distinct cluster of Doppler components near its systemic velocity in addition to the high-velocity lines. At a distance of $\sim 100 \mathrm{Mpc}, 14$ times more distant than NGC 4258, NGC 6323 is a strong candidate for detailed VLBI studies that could ultimately provide a direct measurement of the Hubble constant. Because of the faintness of its masers, a global array of telescopes including the GBT, Effelsberg, and Goldstone in addition to the VLBA would be essential to map the disk.

\section{Summary}

We searched 145 nearby active galaxies for $1.3 \mathrm{~cm} \mathrm{H}_{2} \mathrm{O}$ maser emission and detected 12 galaxies. Although each of these galaxies has been observed but not detected in $\mathrm{H}_{2} \mathrm{O}$ during previous surveys, we attained a fairly high detection rate of $8.3 \%$ due to the improved sensitivity of this survey. Four of the newly discovered masers have spectral profiles consistent with a disk maser and might become useful subjects of followup observations to map the disk, characterize the kinematics, and perhaps make direct measurements of the distances to the host galaxies. Our results indicate that greater than a third of Seyfert 2 galaxies host water megamasers, though the detection rate declines for galaxies beyond $\sim 5000 \mathrm{~km} \mathrm{~s}^{-1}$ due to sensitivity limits. Although Seyfert 2 galaxies are the most likely class of maser host, one of the masers discovered in this survey is in the type 1.5 Seyfert NGC 4151, and another is in the starburst galaxy NGC 2782. We discuss possible reasons for luminous water maser emission in these two galaxies. Our survey demonstrates that the recent improvements in sensitivity and bandwidth warrant more extensive surveys, including type 1 AGNs, starburst galaxies, and apparently normal galaxies.

We would like to extend our appreciation to the GBT operators and the Green Bank staff for support during this program. We thank Don Wells for helpful suggestions concerning analysis techniques, and Ron Maddalena for technical assistance with the GBT. We also thank Bob Garwood and Joe McMullin for their significant contributions to the data analysis system in AIPS++. This research has made use of the NASA/IPAC Extragalactic Database (NED) which is operated by the Jet Propulsion Laboratory, California Institute of Technology, under contract with the National Aeronautics and Space Administration. This research was supported in part by NASA through grant NAG 513065 to the University of Maryland. 
Table 1: $\mathrm{H}_{2} \mathrm{O}$ Masers Detected During the GBT Survey

\begin{tabular}{|c|c|c|c|c|c|c|c|c|}
\hline \multirow[t]{2}{*}{ Source } & \multicolumn{3}{|c|}{$\overline{\alpha_{2000}}$} & " & \multirow{2}{*}{$\begin{array}{r}\mathrm{V}_{L S R}{ }^{\mathrm{a}} \\
\left(\mathrm{km} \mathrm{s}^{-1}\right)\end{array}$} & \multirow{2}{*}{$\begin{array}{c}S_{\text {peak }}^{\mathrm{b}} \\
(\mathrm{mJy})\end{array}$} & \multirow{2}{*}{$\begin{array}{l}\mathrm{L}_{i s o}{ }^{\mathrm{c}} \\
\left(L_{\odot}\right)\end{array}$} & \multirow[t]{2}{*}{ Date of Observation } \\
\hline & $(\mathrm{h}$ & $\mathrm{m} \quad \mathrm{s}$ ) & $\left({ }^{\circ}\right.$ & $1 " 1)$ & & & & \\
\hline NGC 591 & 01 & 13331.2 & +3 & 354006 & $4554 \pm 9$ & 10 & 25 & 31 Jan 2004 \\
\hline Mrk 1066 & 02 & 25958.6 & +3 & 364914 & $3601 \pm 22$ & 48 & 30 & 28 Jan 2004 \\
\hline UGC 3255 & & 50950.2 & +0 & 072900 & $5674 \pm 59$ & 15 & 16 & 6 Mar 2003 \\
\hline Mrk 3 & & 51536.3 & +7 & 710215 & $4009 \pm 6$ & 17 & 11 & 28 Jan 2004 \\
\hline Mrk 78 & & 74241.7 & +6 & 651037 & $11196 \pm 29$ & 28 & 34 & 15 Nov 2003 \\
\hline NGC 2782 & & 91405.1 & +4 & 400649 & $2560 \pm 5$ & 45 & 12 & 14 Jan 2004 \\
\hline NGC 4151 & & 21032.6 & +3 & 392421 & $1002 \pm 3$ & 52 & 0.7 & 28 Feb 2004 \\
\hline NGC 4388 & & 22546.7 & +1 & 123944 & $2521 \pm 4$ & 15 & 12 & 14 Jan 2004 \\
\hline NGC 4922 & & 30125.2 & +2 & 291850 & $7080 \pm 9$ & 36 & 190 & 31 Oct 2003 \\
\hline NGC 5256 & & 33817.2 & +4 & 481632 & $8365 \pm 13$ & 99 & 30 & 4 Nov 2003 \\
\hline NGC 5728 & & 14223.9 & -1 & 171511 & $2796 \pm 8$ & 173 & 88 & 14 Jan 2004 \\
\hline NGC 6323 & & 71318.0 & +4 & 434656 & $7791 \pm 35$ & 41 & 480 & 2 Jun 2003 \\
\hline
\end{tabular}

${ }^{a}$ Recession velocity measured with respect to the local standard of rest. Velocities and uncertainties are from de Vaucouleurs et al. (1991), using HI velocities when available and optical velocities otherwise. For NGC 6323 the optically measured velocity is from Marzke, Huchra \& Geller (1996).

${ }^{b}$ Peak flux density

${ }^{c}$ Inferred isotropic luminosity of the maser emission. 
Table 2: Galaxies Undetected in $\mathrm{H}_{2} \mathrm{O}$ Emission

\begin{tabular}{lllll}
\hline $0152+0622$ & IC 4553 & NGC 334 & NGC 3660 & NGC 5427 \\
$0253-1641$ & II Zw 101 & NGC 404 & NGC 3898 & NGC 5635 \\
$0258-1136$ & Mrk 176 & NGC 600 & NGC 3921 & NGC 5674 \\
$0335+09$ & Mrk 198 & NGC 788 & NGC 3982 & NGC 5675 \\
$0354-1855$ & Mrk 273 & NGC 1144 & NGC 3998 & NGC 5695 \\
$0414+00$ & Mrk 298 & NGC 1167 & NGC 4013 & NGC 5851 \\
$0445-1741$ & Mrk 334 & NGC 1229 & NGC 4036 & NGC 5899 \\
$0446-2349$ & Mrk 359 & NGC 1358 & NGC 4074 & NGC 5929 \\
$07570+2334$ & Mrk 372 & NGC 1365 & NGC 4111 & NGC 5953 \\
$0816+211$ & Mrk 403 & NGC 1409 & NGC 4117 & NGC 6211 \\
$0942+09$ & Mrk 423 & NGC 1410 & NGC 4192 & NGC 6251 \\
$1034+060$ & Mrk 461 & NGC 1667 & NGC 4278 & NGC 6500 \\
1116-2909 & Mrk 477 & NGC 1685 & NGC 4303 & NGC 6764 \\
$1258-3208$ & Mrk 516 & NGC 2110 & NGC 4419 & NGC 6951 \\
1319-162 & Mrk 573 & NGC 2273 & NGC 4450 & NGC 7212 \\
1322+2918 & Mrk 612 & NGC 2377 & NGC 4486 & NGC 7217 \\
1335+39 & Mrk 622 & NGC 2768 & NGC 4501 & NGC 7450 \\
1431-3237 & Mrk 745 & NGC 2841 & NGC 4569 & NGC 7674 \\
$1533+14$ & Mrk 883 & NGC 2911 & NGC 4579 & NGC 7682 \\
1548-0344 & Mrk 917 & NGC 2992 & NGC 4941 & NGC 7743 \\
$2319+09$ & Mrk 937 & NGC 3010b & NGC 5005 & [SP] 55 \\
3C 317 & Mrk 955 & NGC 3031 & NGC 5128 & UGC 3995A \\
Ark 539 & Mrk 1058 & NGC 3185 & NGC 5135 & UGC 6100 \\
Fair 1140 & Mrk 1073 & NGC 3227 & NGC 5195 & UGC 10567 \\
Fair 1149 & Mrk 1098 & NGC 3362 & NGC 5252 & UGC 12056 \\
IC 614 & Mrk 1239 & NGC 3561 & NGC 5283 & \\
IC 694 & Mrk 1388 & NGC 3642 & NGC 5371 & \\
\hline
\end{tabular}



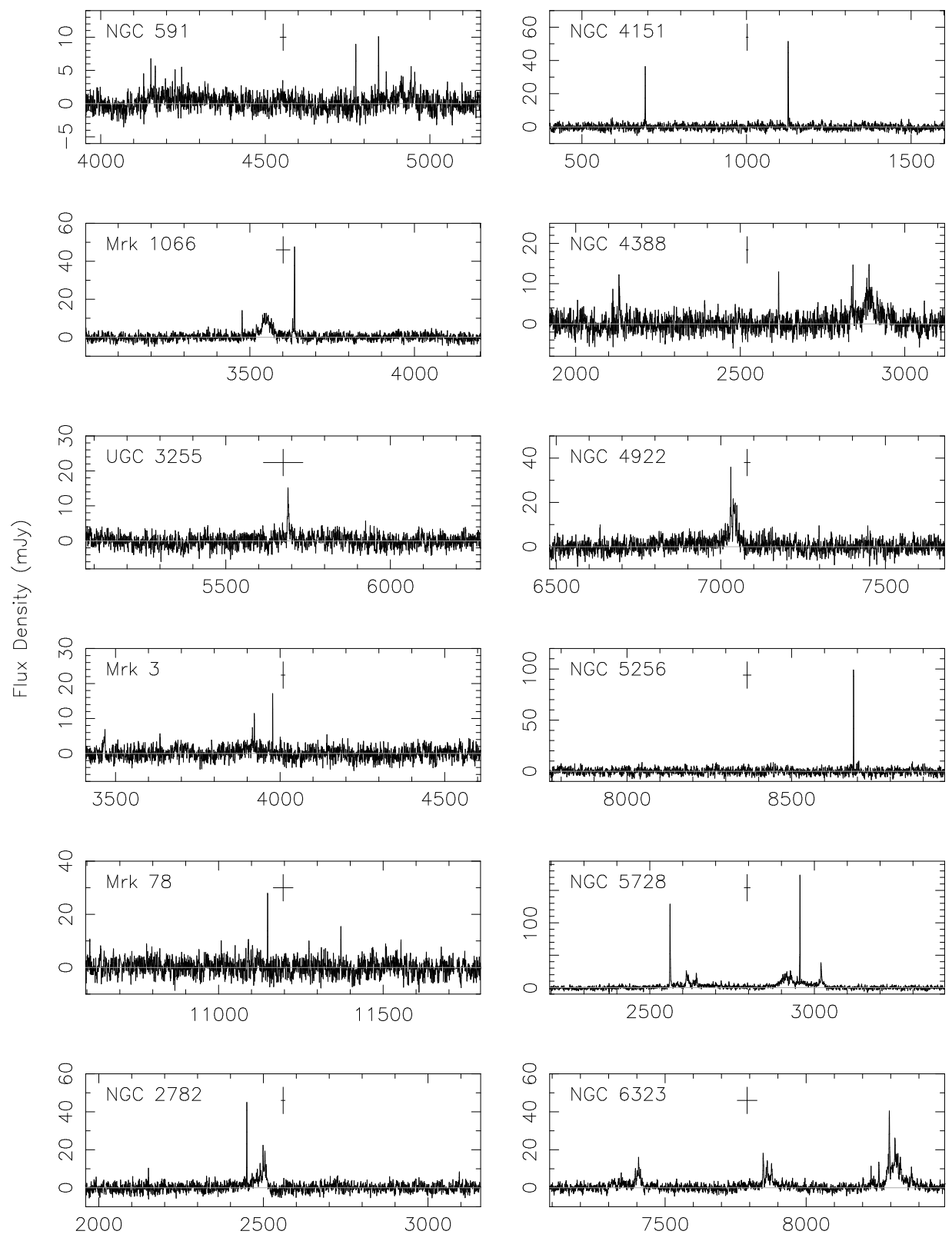

LSR Velocity $(\mathrm{km} / \mathrm{s})$
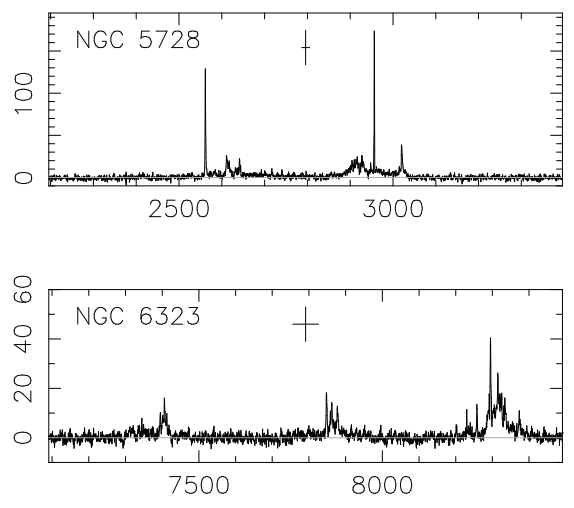

LSR Velocity $(\mathrm{km} / \mathrm{s})$

Fig. 1. - Spectra of $1.3 \mathrm{~cm}$ water maser emission toward the nuclei of 12 galaxies. Each spectrum covers $1200 \mathrm{~km} \mathrm{~s}^{-1}$ except the one toward NGC 6323, which covers $1400 \mathrm{~km} \mathrm{~s}^{-1}$. The systemic velocity of each galaxy and its uncertainty are marked above each spectrum by a cross. Velocities are measured with respect to the LSR and use the optical definition of Doppler shift. 


\section{REFERENCES}

Braatz, J.A., Wilson, A.S., \& Henkel, C. 1996, ApJS, 106, 51

Braatz, J.A., Wilson, A.S., \& Henkel, C. 1997, ApJS, 110, 321

Braatz, J.A., Wilson, A.S., Henkel, C., Gough, R. \& Sinclair, M. 2003, ApJS, 146, 249

Corbin, M.R., Baldwin, J.A. \& Wilson A.S. 1988, ApJ, 334, 584

de Vaucouleurs, G., de Vaucouleurs, A., Corwin, H.G., Buta, R.J., Paturel, G. \& Fouque, P. 1991, Third Reference Catalog of Bright Galaxies, (Berlin:Springer Verlag)

Greenhill, L.J., Henkel, C., Becker, R., Wilson, T.L. \& Wouterloot, J.G.A. 1995a, A\&A, 304, 21

Greenhill, L.J., Jiang, D.R., Moran, J.M., Reid, M.J., Lo, K.Y., \& Claussen, M.J. 1995b, ApJ, 440, 619

Greenhill, L.J., Herrnstein, J.R., Moran, J.M., Menten, K.M. \& Velusamy, T. 1997, ApJ, 486, L15

Greenhill, L.J. 2002, in Cosmic Masers: From Protostars to Blackholes, ed. V. Migenes \& M. J. Reid (San Francisco: IAU Symposium Vol. 206), 381

Greenhill, L.J., Kondratko, P.T., Lovell, J.E.J., Kuiper, T.B.H., Moran, J.M., Jauncey, D.L. \& Baines, G.P. 2003, ApJ, 582, L11

Hagiwara, Y., Diamond, P.J. \& Miyoshi, M. 2002, A\&A, 383, 65

Hagiwara, Y., Diamond, P.J., Miyoshi, M., Rovilos, E. \& Baan, W. 2003, MNRAS, 344, L53

Haschick, A.D., Baan, W.A. \& Peng, E.W. 1994, ApJ, 437, L35

Henkel, C., Braatz, J.A., Greenhill, L.J. \& Wilson, A.S. 2002, A\&A, 394, L23

Henkel, C. \& Braatz, J.A. 2003, Acta Astronomica Sinica Supplement Issue, 44, 55

Herrnstein, J.R., Moran, J.M., Greenhill, L.J., Diamond, P.J., Inoue, M., Nakai, N., Miyoshi, M., Henkel, C. \& Riess, A. 1999, Nature, 400, 539

Humphreys, E.M.L., Argon, A.L., Greenhill, L.J., Moran, J.M. \& Reid, M.J. 2004, to be published in ASP Conference Series on VLBA 10th Anniversary Meeting 
Kauffmann, G., Heckman, T.M., Tremonti, C., Brinchmann, J., Charlot, S., White, S.D.M., Ridgway, S.E., Brinkmann, J., Fukugita, M., Hall, P.B., Ivezic, Z., Richards, G.T. \& Schneider, D.P. 2003, MNRAS, 346, 1055

Kennicutt, R.C., Keel, W.C. \& Blaha, C.A. 1989, AJ, 97, 1022

Kondratko, P.T., Greenhill, L.J., Moran, J.M., Lovell, J.E.J., Jauncey, D.L. \& Kuiper, T.B. 2003, BAAS, 35, 1311

Maloney, P.R. 2002, PASA, 19, 401

Marzke, R.O., Huchra, J.P. \& Geller, M.J. 1996, AJ, 112, 1803

Miyoshi, M., Moran, J., Herrnstein, J., Greenhill, L., Nakai, N., Diamond, P., \& Inoue, M. 1995, Nature, 373, 127

Osterbrock, D.E. \& Koski, A.T. 1976, MNRAS, 176, 61

Peck, A.B., Tarchi, A., Henkel, C., Nagar, N.M., Braatz, J.A. \& Moscadelli, L. 2003, "The Astrochemistry of External Galaxies", 25th meeting of the IAU, Joint Discussion 21, Sydney, Australia

Pogge, R.W. 1988, ApJ, 332, 702

Schulz, H., Komossa, S., Berghofer, T.W. \& Boer, B. 1998, A\&A, 330, 823

Sergeev, V.I., Pronik, S.G. \& Sergeeva, E.A. 2001, ApJ, 554, 245

Seyfert, C. 1943, ApJ, 97, 28

Taylor, G.B., Peck, A.B., Henkel, C., Falcke, H., Mundell, C.G., O’Dea, C.P., Baum, S.A. \& Gallimore, J.F. 2002, ApJ, 574, 88

Turner, T.J., George, I.M., Nandra, K. \& Mushotsky, R.F. 1997, ApJS, 113, 23

Véron, P., Gonçalves, A.C. \& Véron-Cetty, M.-P. 1997, A\&A, 319, 52

Weaver, K.A., Yaqoob, T., Holt, S.S., Mushotzky, R.F., Matsuoka, M. \& Yamauchi, M. 1994, ApJ, 436, L27

Wilson, A.S., Braatz, J.A., Heckman, T.M., Krolik, J.H. \& Miley, G.K. 1993, ApJ, 419, L61 